\title{
Smart Composites With Short Ferromagnetic Microwires for Microwave Applications
}

\author{
F. X. Qin ${ }^{1,2}$, H. X. Peng ${ }^{1}$, L. V. Panina ${ }^{3}$, M. Ipatov ${ }^{4}$, V. Zhukova ${ }^{4}$, A. Zhukov ${ }^{4,5}$, and J. Gonzalez ${ }^{4}$ \\ ${ }^{1}$ Advanced Composite Center for Innovation and Science, Department of Aerospace Engineering, University of Bristol, \\ Bristol BS8 1TR, U.K. \\ ${ }^{2}$ Lab-STICC, Université de Bretagne Occidentale, CS 93837, 29238 Brest Cedex 3, France \\ ${ }^{3}$ School of Computing, Communication and Electronics, University of Plymouth, Drake Circus, Plymouth, Devon PL4 8AA, U.K. \\ ${ }^{4}$ Dpto. Física de Materiales, Fac. Químicas, UPV/EHU, 20018 San Sebastián, Spain \\ ${ }^{5}$ IKERBASQUE, Basque Foundation for Science, 48011 Bilbao, Spain
}

\begin{abstract}
Smart composites with short-cut $\mathrm{Co}_{68.7} \mathrm{Fe}_{4} \mathrm{Ni}_{1} \mathrm{~B}_{13} \mathrm{Si}_{11} \mathrm{Mo}_{2.3}$ microwires were prepared and studied in terms of their microwave tunable properties. It is shown that the frequency dependence of effective permittivity relaxes with the application of magnetic field till around the anisotropy field of the microwire due to the increase of internal losses. There exists a significant field tunable effect in the transmission and reflection spectra, featured as a resonance-relaxation transformation; a step-like shift of reflection phase was also observed with increasing applied magnetic field, which can be exploited especially for the sensing applications such as field/stress monitoring. Notably, with increasing microwire concentration from $0.06 \mathrm{~cm}^{-2}$ to $0.24 \mathrm{~cm}^{-2}$, the microwave absorption is more than doubled; the reflection phase shift corresponding to the magnetic field change from 500 to $1000 \mathrm{~A} / \mathrm{m}$ is also increased from 1.2 to 1.3 . These results indicate that the developed short-wire composites have the potential for microwave absorption and remote sensing applications.
\end{abstract}

Index Terms-Effective permittivity, ferromagnetic amorphous wires, smart materials, tunable composites.

\section{INTRODUCTION}

$\mathbf{M}$ ULTIFUNCTIONAL composites containing microscale or nanoscale fillers have been of much interest to the scientific community in terms of their electromagnetic properties for microwave applications such as tunable microwave devices [1] and remote interrogated sensors [2]. Particularly of interest is the possibility of manipulating the electromagnetic parameters of the composites through the external stimuli such as magnetic field, stress and temperature; such tunable property has been demonstrated in a smart microwire polymer composite, which contains long continuous ferromagnetic wires [3]. This kind of composite presents very good adjustability, which is highly needed for the reconfigurable device for engineering applications of pressing needs such as microwave non-destructive tests and structural health monitoring [1]. As a follow-up study, this work targets on the short-wire composites, i.e., the composite containing short wire pieces rather than continuous wires.

Short wire inclusions in the composite matrix, as elementary dipole scatterers, can be polarized when they are excited by the microwave. In the presence of an axial magnetic field or stress, significant impedance variation will occur in that the magnetization is rotated away from the circumferential anisotropy direction. This then will lead to a considerable change in the current distribution along the wire and hence in the induced dipole moment. Thus, the average dielectric response from the composite

Manuscript received February 18, 2011; revised May 16, 2011; accepted May 17, 2011. Date of current version September 23, 2011. Corresponding author: F. X. Qin (e-mail: Faxiang.Qin@bristol.ac.uk; Faxiang.Qin@univ-brest.fr).

Color versions of one or more of the figures in this paper are available online at http://ieeexplore.ieee.org.

Digital Object Identifier 10.1109/TMAG.2011.2157663 in the free space characterized by the effective permittivity will also show significant variations, often featured as a resonance to a relaxation dispersion transformation. Mathematically, the effective permittivity $\varepsilon_{\text {eff }}$ can be expressed as [1]

$$
\varepsilon_{\text {eff }}=\varepsilon_{m}+4 \pi p\langle\alpha\rangle
$$

where $\varepsilon_{m}$ is the matrix permittivity, $p$ the volume concentration and $\langle\alpha\rangle$ the average polarization that can be given by [1]

$$
\begin{aligned}
\langle\alpha\rangle & =\frac{1}{2 \pi \ln (l / a)(K a)^{2}}\left(\frac{2}{K l} \tan (K l / 2)-1\right) \\
K & =k\left(1+\frac{i c \zeta_{z z}}{\omega a \ln (l / a)}\right)^{1 / 2}, \quad k=\omega \sqrt{\varepsilon_{m}} / c
\end{aligned}
$$

where $l$ denotes the length, $a$ is the wire diameter, $\omega$ is the angle frequency, $c$ is the light speed, and $\zeta_{\mathrm{zz}}$ is the wire surface impedance. According to (1)-(3), the correlation between the impedance change of the single wire and the collective dielectric response of the wire composite is established.

\section{EXPERIMENTAL DETAILS}

We fabricated short-wire composites with two different wire area concentrations $n_{c}=0.06$ and $0.24 \mathrm{~cm}^{-2}$. Here $n_{c}$ is defined as the number of wires per unit area. Specifically, $\mathrm{Co}_{68.7} \mathrm{Fe}_{4} \mathrm{Ni}_{1} \mathrm{~B}_{13} \mathrm{Si}_{11} \mathrm{Mo}_{2.3}$ glass-covered magnetic microwires with a metallic core diameter of $8.0 \mu \mathrm{m}$ and a glass layer thickness of $2.4 \mu \mathrm{m}$ were prepared by a modified Taylor-Ulitovskiy process [4]. The impedance was calculated through the complex reflection coefficient, which was measured by the microwave waveguide method. The microwire was fixed between two auxiliary waveguide sections parallel to the narrow wall. Loads up to $3 \mathrm{~g}$ were applied to the microwire along the wire axis. Following the procedure detailed in our 
previous work [5], the short-wire composites were prepared by embedding the $50 \mathrm{~mm}$ long microwires periodically with different spacing into E-glass 913 prepregs, as schematically shown in Fig. 1. The S-parameters were measured in the frequency range of $0.9-17 \mathrm{GHz}$ in the presence of external field ranging up to $3 \mathrm{kA} / \mathrm{m}$ applied through a plane coil with turns perpendicular to the electrical field in the incident wave. The effective permittivity spectra were deduced from S-parameters using the Reflection/Transmission Epsilon Fast Model. More details can be found in [6].

\section{RESUltS AND DisCUSSION}

The studied Co-based amorphous wire is an excellent soft magnetic material. It possesses a circumferential anisotropy with the effective anisotropy field of about $500 \mathrm{~A} / \mathrm{m}$ [Fig. 2(a)]. The microwave impedance of the wire is shown in Fig. 2(b). It can be seen the impedance changes sensitively with external magnetic field or stress. In terms of domain structure, the Co-rich wire consists of an inner core with axial anisotropy and an outer shell with circumferential anisotropy. The ratio of the inner core radius to the whole metallic core radius can be approximated as the ratio of remnant magnetization to saturation magnetization [7], therefore, the outer shell occupies a large fraction of whole metallic core. Consequently, the axial magnetization process is dominated by the domain rotation. This makes the magnetization process very sensitive to the stress, in that the magnetization angle is sensitive to the stress, especially in the presence of a dc biased field of the order of anisotropy field along the wire axis [2].

It is worth mentioning that, GMI properties of the wire are dominated by the magnetoelastic energy $\left(K_{m e}\right)$ contributed by the negative magnetostriction constant $\left(\lambda_{s}\right)$ and frozen-in stress $\left(\sigma_{i}\right)$, mainly caused by the difference of thermal coefficients of glass coat and metallic core, formed in the fabrication process: $K_{m e} \approx 3 / 2 \lambda_{s} \sigma_{i}$, where $\lambda_{\mathrm{s}}$ is external stress $(\sigma)$ dependent: $\lambda_{s}=\left(\mu_{0} M_{s} / 3\right)\left(d H_{k} / d \sigma\right)$ [8], [9]. Thus, a delicate change of composition and geometry of the wire as well as the external stimuli such as stress could result in a significant change of the magnetic behavior of the wires. This lays the foundation for tuning the collective response of the wire composites by changing the local magnetic properties for a single wire.

Fig. 3 shows the dispersion of effective permittivity for a short-wire composite with the parameters $\left(l, a, n_{c}\right)$ detailed in the graph. The increasing external magnetic field monotonously depreciated $\varepsilon^{\prime \prime}$, indicating a drop of microwave attenuation. The values of $\varepsilon^{\prime}$ and $\varepsilon^{\prime \prime}$ are rather small due to the low concentration of the microwires (Fig. 3(a)). With $n_{c}$ increased by 4 times, a significant increase of $\varepsilon^{\prime}$ and $\varepsilon^{\prime \prime}$ occurs as shown in Fig. 3(b). The tunability of the $\varepsilon^{\prime}$ (variation of $\varepsilon^{\prime}$ to that of corresponding magnetic field) is also increased by about 10 times. In particular, in the case of the $n_{c}=0.06$ composite, $\varepsilon^{\prime \prime}$ is smaller than $\varepsilon^{\prime}$, which means the dielectric loss factor is smaller than unity. As $n_{c}$ increases to $0.24 \mathrm{~cm}^{-2}, \varepsilon^{\prime \prime}$ increases notably to such an extent that its value is higher than $\varepsilon^{\prime}$, which indicates the dielectric loss factor is larger than unity. As the dispersion is anomalous, the transformation of resonance to relaxation can be inferred from the frequency evolution of these curves. The anisotropy field can be used as a critical value to distinguish the frequency

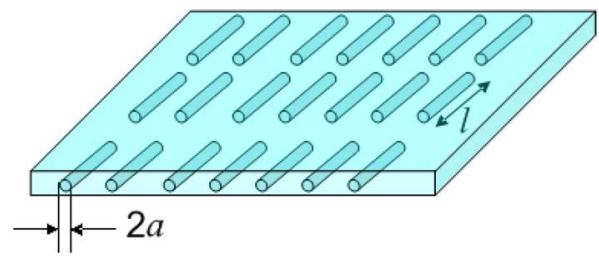

Fig. 1. Schematic of the short-wire composite. $l$ indicates the wire length, $a$ the wire radius.
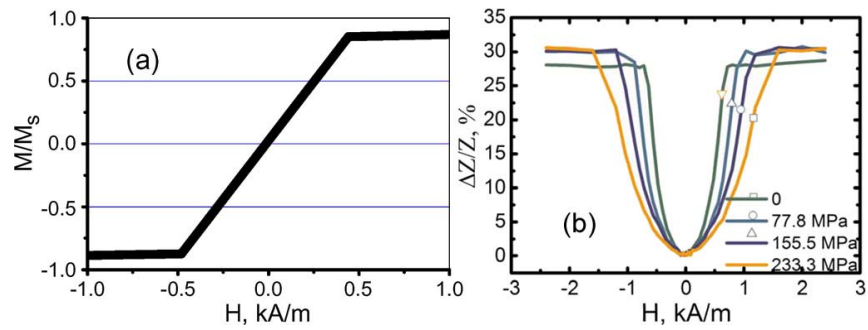

Fig. 2. Axial magnetic field dependence of (a) magnetization curve and (b) magnoimpedance ratio profiles in the presence of varying stresses at $9 \mathrm{GHz}$ for $\mathrm{Co}_{68.7} \mathrm{Fe}_{4} \mathrm{Ni}_{1} \mathrm{~B}_{13} \mathrm{Si}_{11} \mathrm{Mo}_{2.3}$ glass-covered magnetic microwires with a metallic core diameter of $8.0 \mu \mathrm{m}$ and a glass layer thickness of $2.4 \mu \mathrm{m}$.
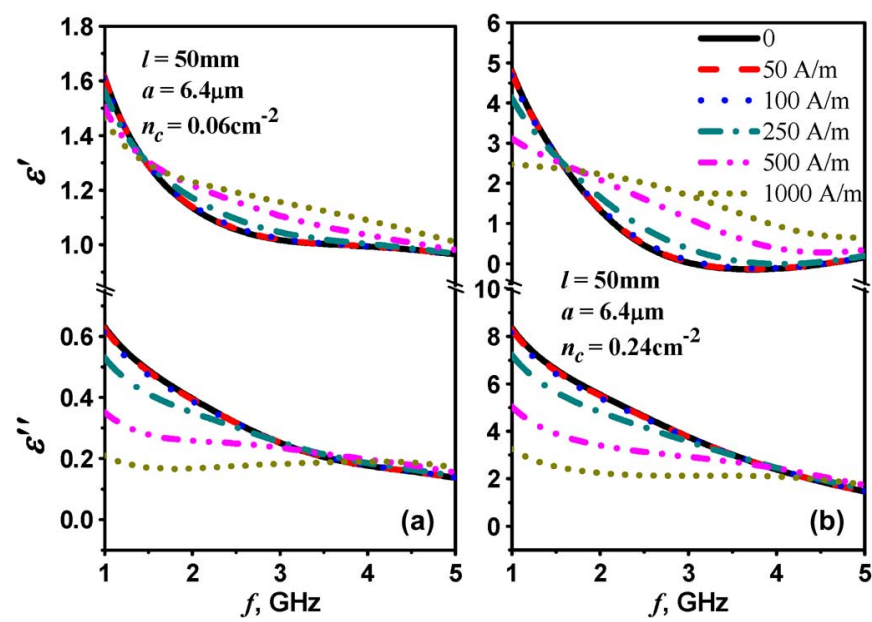

Fig. 3. Effective permittivity spectra of the (a) $n_{c}=0.06 \mathrm{~cm}^{-2}$ and (b) $n_{c}=$ $0.24 \mathrm{~cm}^{-2}$ short-wire composite in the presence of varying dc magnetic field. The material parameters are given in the graph: $l$ is the wire length, $a$ is the wire radius, $n_{c}$ is the ratio of wire number to the area containing them.

dependence of $\varepsilon^{\prime}$ at studied frequency range (1-5 GHz). The same trend is also observed in the frequency plots of $\varepsilon^{\prime \prime}$. It can be explained as follows. When $\mathrm{H}_{\mathrm{ex}}<\mathrm{H}_{\mathrm{k}}$, the impedance is increased with $\mathrm{H}_{\mathrm{ex}}$. Therefore, the internal loss increases and the relaxation dispersion occur. The relaxation behavior is fully achieved when $\mathrm{H}_{\mathrm{ex}}=\mathrm{H}_{\mathrm{k}}$, whereby the impedance reaches a maximum. Further increase of $\mathrm{H}_{\mathrm{ex}}$ results in a reverse trend. Note that the dielectric response to the magnetic field is not seen until the field is $250 \mathrm{~A} / \mathrm{m}$; this can be attributed to the relative insensitivity of magnetoimpedance for this range of magnetic field.

As with the complex permittivity spectra, the transmission amplitude is found to increase with increasing field alongside resonance-relaxation change, as seen in Fig. 4(a). As A + T + $\mathrm{R}=1$, where $\mathrm{A}$ is absorption, $\mathrm{T}$ is transmission and $\mathrm{R}$ is reflection. The effective absorption can be expressed as $1-T /(1-R)$. 

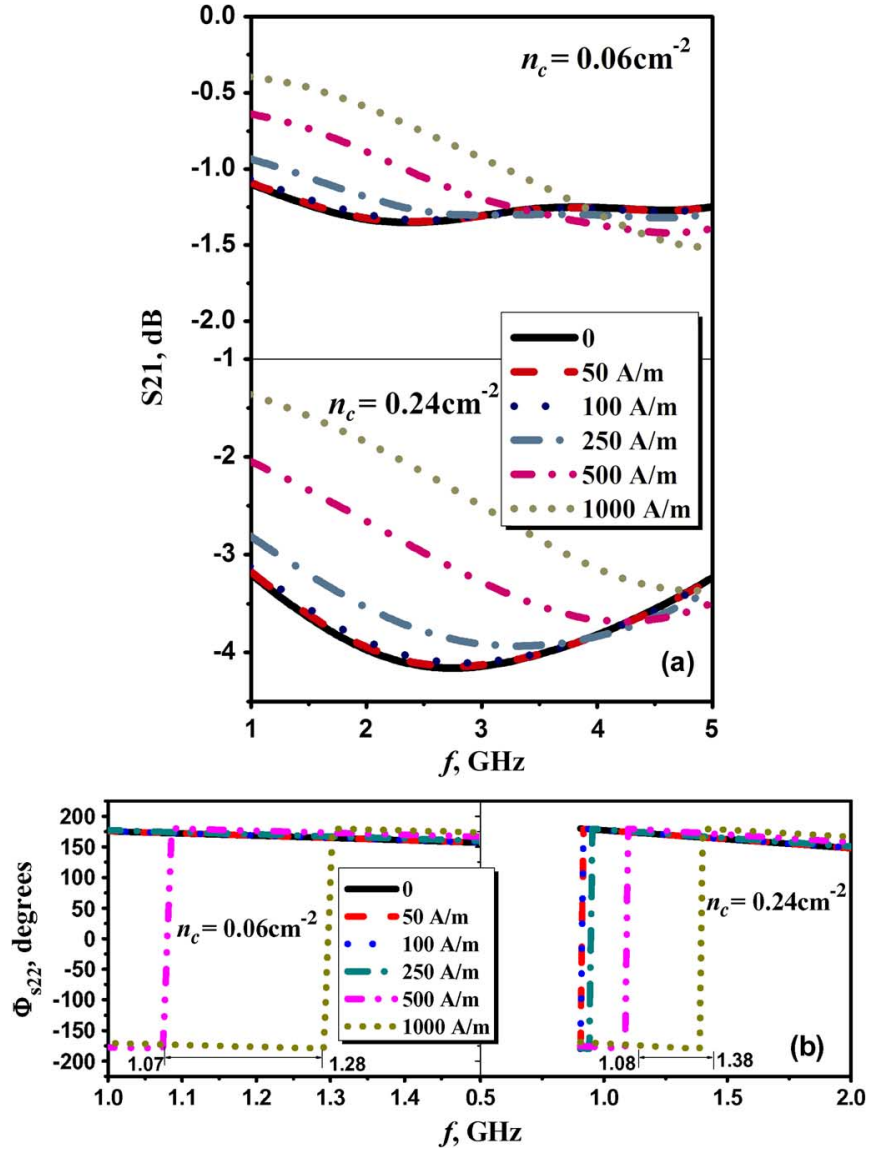

Fig. 4. Measured transmission $\mathrm{S} 21$ (a) and reflection phase $\Phi_{22}$ spectra (b) of the two short-wire composite of different $n_{c}$ with the magnetic field as a parameter.

We can then obtain that with increasing $n_{c}$ the effective absorption increases from $20 \%$ to $47 \%$ by c.a. 2.3 times for $\mathrm{H}_{\mathrm{ex}}=0$ at $1-5 \mathrm{GHz}$; the tunability of transmission is also increased by about 2 times. The maximum transmission (absorption) shifts to higher frequency. A slight increase of step-like phase shift from 1.2 for $\mathrm{n}_{\mathrm{c}}=0.06 \mathrm{~cm}^{-2}$ to 1.3 for $\mathrm{n}_{\mathrm{c}}=0.24 \mathrm{~cm}^{-2}$ is observed with the field increasing from 500 to $1000 \mathrm{~A} / \mathrm{m}$ [Fig. 4(b)]; such a feature can be exploited for magnetic field or stress sensing applications.

According to (1), increasing $n_{c}$ or wire amount improves the effective polarization and hence the permittivity and its tunability. Also, the long-range dipolar interactions between the wires can be enhanced with decreasing wire spacing, giving rise to increasing anisotropy and hence the magnetic permeability [10]. As a result, reflection and absorption increase so that transmission amplitude is increased. Their tunability is also affected. The pronounced increase of the absorption can be accounted for by the considerable increase of dielectric loss factor. The absorption is attributed to the dielectric loss and magnetic loss in the present case. Oscillating current is generated in the microwires by incident microwave, which results in the occurrence of electric dipole resonance (EDR). Thus, part of microwave electromagnetic energy will be dissipated. Note that this is different from the case of continuous-wire composites, whereby the electrons is confined within the wires and result in a plasma characteristics of electric polarization [6]. Meanwhile, the microwave magnetic component in the radial direction results in the precession of circumferential magnetic moment, which induces natural ferromagnetic resonance (NFMR) [11]. Although the skin effect is strong at measured frequencies, the microwave magnetic field can still penetrate the outer layer and consume part of the microwave magnetic energy. Clearly, the fine size of the wires is important to achieve better absorption. The maximum absorption shifting to higher frequency can be explained by the increasing EDR and NFMR resonance due to the enhanced dipolar interactions. As such, it can be predicted that with further increase of wire concentration, the absorption can be further improved. Compared to the continuous-wire composites, it has been shown in our previous study that the short-wire composites can achieve better impedance match [12], indicating a likely larger microwave absorption. Also, in terms of manufacture, although they prove to be more complex than continuous-wire composites, it is much easier to realize cost-effective mass production of them to meet large-scale engineering applications than, for example, nanocomposites with nanoparticles filled carbon nanotubes (CNTs), which involves a limited quantity of filled CNTs with nanoparticles by complex chemical methods and notoriously difficult dispersion of CNTs in the polymer (see, e.g., [13], [14]). Therefore, the short-wire composites may find wide use in microwave absorption, which is a critical issue in the aeronautical and electrical industry.

It should be noted that, usually, the microwave absorption of planar samples is tested in the spacious microwave anechoic room. The sample is attached onto the metallic plate so that the transmission is eliminated and reflection loss can then be used to characterize the microwave absorption. Common practice is to set $10 \mathrm{~dB}$ as the limit of effective absorption. The maximum absorption and absorption bandwidth also need to be carefully evaluated for practical applications. In this regard, the results presented here cannot serve as handbook data for one to refer to, but it demonstrates the following advantageous potentials of microwire composites: (i) Tunable absorption (including maximum absorption, absorption bandwidth) can be achieved by the external stimuli: the maximum absorption could vary with the loss factor, which is dependent on the applied magnetic field, stress or temperature; it will also shift the frequency where maximum absorption occurs, as FMR can be controlled, for example, by a weak magnetic bias. By optimizing the system, a microwave tunable selective surface would be approachable. (ii) By changing the local properties of the wires such as wire length, metal-to-glass ratio of wires or by performing, for example, annealing on the wires, the magnetic behavior will be modified and the absorption characteristics can accordingly be optimized to meet specific application requirements. Relevant results have been reported on the polymer composite containing randomly oriented wires [11], [15]. We should also be clear that ideally the best absorption can be achieved by orienting the wires along the direction of electrical field vector so that any depolarization effect could be minimized. But in practice, if without enough knowledge of the source of electromagnetic wave, the randomly oriented wires would be preferred. Also, another plausible solution is to make multiplayer of composites containing parallel wires of different orientation for each layer, 
in analogy to the quasi-isotropic fibre reinforced composites. This will be one of the subjects of interest for our future work.

\section{CONCLUSION}

We prepared short-wire composites containing periodically arranged glass-coated ferromagnetic microwires and studied the microwave field tunable effect as well as the absorption properties. It has been shown that with increasing wire concentration, dielectric loss factor is increased markedly in relative to unity due to enhanced electric dipole resonance, and the maximum absorption is increased by over 2 times and shifts to higher frequency at $1-5 \mathrm{GHz}$ due to the long-range dipolar interactions between the wires. The tunable field effects are identified as a resonance-relaxation transformation in the transmission/reflection spectra as well as a step-like shift of reflection phase in the reflection phase spectra. Their tunability is also dependent on the wire concentration. All these results suggest that the developed short-wire composites are promising for microwave absorption and remote sensing applications.

\section{ACKNOWLEDGMENT}

F. X. Qin was supported through Overseas Research Students Awards Scheme and University of Bristol Postgraduate Student Scholarship. This work was supported by EU ERA-NET programme under project "SoMaMicSens" (MANUNET-2010-Basque-3), by EU under FP7 "EM-safety" project, and by Spanish Ministry of Science and Innovation under Project MAT2010-18914.

\section{REFERENCES}

[1] D. P. Makhnovskiy and L. V. Panina, "Field and stress tunable microwave composite materials based on ferromagnetic wires," in Progress in Ferromagnetism Research, V. N. Murray, Ed. Hauppauge, NY: Nova Science, 2005.
[2] K.-H. Shin, M. Inoue, and K.-I. Arai, "Strain sensitivity of highly magnetostrictive amorphous films for use in microstrain sensors," J. Appl. Phys.., vol. 85, pp. 5465-5467, 1999.

[3] F. X. Qin, N. Pankratov, H. X. Peng, M. H. Phan, L. V. Panina, M. Ipatov, V. Zhukova, A. Zhukov, and J. Gonzalez, "Novel magnetic microwires-embedded composites for structural health monitoring applications," J. Appl. Phys., vol. 107, pp. 09A314-09A314-3, 2010.

[4] V. S. Larin, A. V. Torcunov, A. Zhukov, J. Gonzalez, M. Vazquez, and L. Panina, "Preparation and properties of glass-coated microwires," $J$. Magn. Magn. Mater., vol. 249, pp. 39-45, 2002.

[5] H. X. Peng, F. X. Qin, M. H. Phan, J. Tang, L. V. Panina, M. Ipatov, V. Zhukova, A. Zhukov, and J. Gonzalez, "Co-based magnetic microwire and field-tunable multifunctional macro-composites," J. NonCryst. Solids, vol. 355, pp. 1380-1386, 2009.

[6] D. P. Makhnovskiy, L. V. Panina, C. Garcia, A. P. Zhukov, and J. Gonzalez, "Experimental demonstration of tunable scattering spectra at microwave frequencies in composite media containing $\mathrm{CoFeCrSiB}$ glasscoated amorphous ferromagnetic wires and comparison with theory," Phys. Rev. B: Condens. Matter, vol. 74, p. 064205, 2006.

[7] M. Vázquez and A. P. Zhukov, "Magnetic properties of glass-coated amorphous and nanocrystalline microwires," J. Magn. Magn. Mater., vol. 160, pp. 223-228, 1996.

[8] A. Zhukov, M. Ipatov, J. Gonzalez, J. M. Blanco, and V. Zhukova, "Recent advances in studies of magnetically soft amorphous microwires," J. Magn. Magn. Mater., vol. 321, pp. 822-825, 2009.

[9] M.-H. Phan and H.-X. Peng, "Giant magnetoimpedance materials: Fundamentals and applications," Prog. Mater Sci., vol. 53, pp. 323-420, Feb. 2008.

[10] E. H. C. P. Sinnecker, F. S. de Menezes, L. C. Sampaio, M. Knobel, and M. Vázquez, "Tailoring coercivity in an array of glass-coated microwires," J. Magn. Magn. Mater., vol. 226-230, pp. 1467-1469, 2001.

[11] P. Marin, D. Cortina, and A. Hernando, "Electromagnetic wave absorbing material based on magnetic microwires," IEEE Trans. Magn., vol. 44, pp. 3934-3937, 2008.

[12] F. X. Qin, H. X. Peng, N. Pankratov, M. H. Phan, L. V. Panina, M. Ipatov, V. Zhukova, A. Zhukov, and J. Gonzalez, "Exceptional EMI shielding properties of ferromagnetic microwires enabled polymer composites," J. Appl. Phys., vol. 108, p. 044510, 2010.

[13] P. Susmita et al., "Carbon nanostraws: Nanotubes filled with superparamagnetic nanoparticles," Nanotechnology, vol. 20, p. 485604, 2009.

[14] E. T. Thostenson, C. Li, and T.-W. Chou, "Nanocomposites in context," Compos. Sci. Technol., vol. 65, pp. 491-516, 2005.

[15] Z. Zhang, C. Wang, Y. Zhang, and J. Xie, "Microwave absorbing properties of composites filled with glass-coated Fe69Co10Si8B13 amorphous microwire," Mater. Sci. Eng. B, vol. 175, pp. 233-237, 2010. 